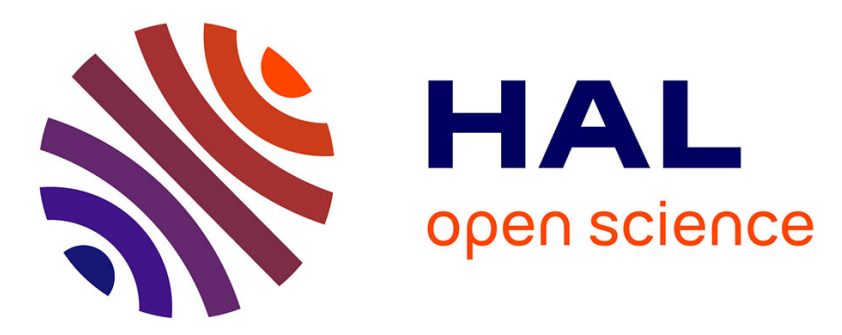

\title{
Improved language performance in Alzheimer disease following brain stimulation
}

\author{
Maria Cotelli, Marco Calabria, Rosa Manenti, Sandra Rosini, Orazio Zanetti, \\ Stefano F Cappa, Carlo Miniussi
}

\section{- To cite this version:}

Maria Cotelli, Marco Calabria, Rosa Manenti, Sandra Rosini, Orazio Zanetti, et al.. Improved language performance in Alzheimer disease following brain stimulation. Journal of Neurology, Neurosurgery and Psychiatry, 2010, 82 (7), pp.794. 10.1136/jnnp.2009.197848 . hal-00600749

\section{HAL Id: hal-00600749 \\ https://hal.science/hal-00600749}

Submitted on 16 Jun 2011

HAL is a multi-disciplinary open access archive for the deposit and dissemination of scientific research documents, whether they are published or not. The documents may come from teaching and research institutions in France or abroad, or from public or private research centers.
L'archive ouverte pluridisciplinaire HAL, est destinée au dépôt et à la diffusion de documents scientifiques de niveau recherche, publiés ou non, émanant des établissements d'enseignement et de recherche français ou étrangers, des laboratoires publics ou privés. 


\section{Improved language performance in Alzheimer disease following brain stimulation}

Maria Cotelli ${ }^{1}$, Marco Calabria ${ }^{1,2}$, Rosa Manenti ${ }^{1}$, Sandra Rosini ${ }^{1}$, Orazio Zanetti ${ }^{1}$,

Stefano F. Cappa ${ }^{3}$ and Carlo Miniussi ${ }^{1,4}$

${ }^{1}$ IRCCS San Giovanni di Dio Fatebenefratelli, Brescia, Italy

${ }^{2}$ Department of General Psychology, University of Padua, Padua, Italy

${ }^{3}$ Vita-Salute University and San Raffaele Scientific Institute \& National Neuroscience

Institute, Milan, Italy

${ }^{4}$ Department of Biomedical Sciences and Biotechnologies \& National Neuroscience

Institute, University of Brescia, Brescia Italy

Correspondence to:

Maria Cotelli, PhD

IRCCS San Giovanni di Dio Fatebenefratelli

Via Piastroni, 4

25125 Brescia Italy

e-mail: mcotelli@fatebenefratelli.it

Phone: +39 0303501593

Fax: +390303501513

Keywords: Neurorehabilitation, rTMS, sentence comprehension, language, transcranial magnetic stimulation

Total Word Count: 1715

Licence for Publication: The Corresponding Author has the right to grant on behalf of all authors and does grant on behalf of all authors, an exclusive license on a worldwide basis to the BMJ Publishing Group to permit this article (if accepted) to be published in JNNP and any other BMJPGL products and sublicenses such use and exploit all subsidiary rights, as set out in our licence.

Competing Interest: None declared.

Consent Form. Since this article does not contain personal medical information about an identifiable living individual we do not include consent forms. 


\section{Abstract}

Objectives: Repetitive Transcranial Magnetic Stimulation (rTMS) has been proposed as a possible treatment for the cognitive deficits associated with Alzheimer Disease (AD). The aim of this study was to assess the long-term effects, on cognitive performance, of rTMS applied to the left dorsolateral prefrontal cortex (DLPFC) in AD patients.

Methods: Ten AD patients were randomly assigned to one of two study groups. Multiplebaseline design was used.The first group underwent a four-week real rTMS stimulation protocol, while the second underwent a two-week placebo treatment, followed by two weeks of real rTMS stimulation. Each session consisted of the application of rhythmic high-frequency rTMS over the DLPFC for 25 minutes. Sessions occurred once daily, five days/week.

The main analyzed outcome was the change in cognitive test performance at two and four weeks after rTMS treatment initiation, with a follow-up performed eight weeks after the end of rTMS, in comparison to baseline performance.

Results: A significant difference was found between groups over sessions in terms of the percentage of correct responses of auditory sentence comprehension. Only real treatment induced an improvement in performance with respect to baseline or placebo. Moreover, both groups showed a lasting effect on the improved performance eight weeks after the end of treatment.

Conclusion: Our findings provide initial evidence for the persistent beneficial effects of rTMS on sentence comprehension in AD patients. Rhythmic rTMS, in conjunction with other therapeutic interventions, may represent a novel approach to the treatment of language dysfunction in AD patients. 


\section{Introduction}

Alzheimer's Disease (AD) is a progressive disorder that impacts memory, language and several other cognitive functions. Given the limited effectiveness of pharmacological treatments, non-pharmacological interventions in AD have gained attention in recent years, and there are currently many different approaches under study, ranging from multistrategy approaches to cognitive training ${ }^{1}$.

Despite the potential therapeutic impact of the non-pharmacological approaches, the neural mechanisms underlying the beneficial effects of behavioral interventions remain largely unknown. Functional neuroimaging studies have shown that rehabilitation in patients with developmental and acquired cerebral damage may lead to functional cortical reorganization, a process mediated by activity-dependent plasticity mechanisms ${ }^{2}$. These "plastic" mechanisms may also play a role in the aging brain and in $\mathrm{AD}^{3}$.

In recent years, new techniques for studying the human brain that allow for the noninvasive neurostimulation have emerged. Repetitive Transcranial Magnetic Stimulation (rTMS) is a technique that delivers several magnetic pulses in rapid sequence up to frequencies of $100 \mathrm{~Hz}$. rTMS can modulate neuronal activity, with the exact effects depending on the stimulation frequency (i.e., $\leq 1 \mathrm{~Hz}$ stimulation results in inhibition, while $\geq 5 \mathrm{~Hz}$ stimulation mostly leads to excitation). There have been no studies to date that have explored the long-term effects of rhythmic off-line rTMS in AD patients. Therefore, the main purpose of the present study was to investigate whether the application of highfrequency rhythmic rTMS, for 2 or 4 weeks, to the left dorsolateral prefrontal cortex (DLPFC) resulted in cognitive improvements ${ }^{4}$ in patients with AD. More specifically, we hypothesized that this type of stimulation may lead to improved language performance i.e., production and/or comprehension. Such prediction comes from a previous work on naming in $A D$ patients ${ }^{5,6}$. A possible effect on sentence comprehension was predicted on the 
basis of a study in young normal subjects ${ }^{7}$, which provided direct evidence of DLPFC involvement in sentence comprehension.

In addition, an important goal of the present study was to verify whether the cognitive benefits, previously recorded solely during on-line rTMS, might persist after the end of the stimulation. We adopted a multiple-baseline design, comparing the stimulation effects with a placebo condition (sham-stimulation) during the first two weeks of treatment. This phase was followed by two weeks of rTMS stimulation in all patients, in order to evaluate whether a longer rTMS application (four vs. two weeks) would further improve the expected benefits in the patient's performance. Finally, we assessed the persistence of the effects eight weeks after the end of the treatment (Figure 1).

\section{Subjects and Methods}

\section{Participants}

Outpatients $(n=10)$ diagnosed as having probable moderate AD, according to the NINCDS-ADRDA ${ }^{8}$ criteria, were enrolled.

Patients with potentially confounding neurological and psychiatric disorders, epilepsy clinically recorded hearing or vision impairment, or with a history of alcohol abuse, psychosis or major depression were not included in the study. All patients had been on a stable dose of cholinesterase inhibitor (donepezil or rivastigmine) for at least six months prior to the onset of the study. The use of drugs with anticolinergic properties was used as an exclusion criterion.

\section{rTMS}

Patients were randomly assigned to one of the two groups: i) Real-Real group (RR), in which the patients received four weeks of rTMS stimulation of the DLPFC; ii) PlaceboReal group (PR), in which patients received DLPFC placebo stimulation during the first two 
weeks followed by two weeks of real stimulation (Figure 1A). Each week of rTMS treatment consisted of 5 sessions ( 25 minutes each, one per day). rTMS was delivered by a Magstim unit featuring a double $70 \mathrm{~mm}$ cooled coil. Before starting the rTMS treatment, the motor excitability stimulation threshold was established for each subject (51.56 $\pm 5.9 \%)$. The stimulation intensity used during the experiment was set to $100 \%$ of each subject's motor threshold. Trains of rhythmic high-frequency $(20 \mathrm{~Hz})$ rTMS were delivered in short periods (2sec-duration) separated by longer periods $(28 \mathrm{sec}$ ) of no stimulation, for each daily session. The total number of pulses for each session was 2000 (40 stimuli/train, 50 trains). These parameters are consistent with safety recommendations for TTMS $^{9}$. Furthermore, all participants tolerated rTMS well and did not report any adverse effects. In the placebo condition, a sham coil was used ${ }^{10}$.

Figure-1

We localized the target areas using the SofTaxic neuronavigator system (www.emsmedical.net) on an MRI template. Based on these estimated MRIs, the average location of the stimulating points was centered on Talairach coordinates $X=-35, Y=24$, $Z=48$, corresponding to the DLPFC (Brodmann Area 8/9). We chose to stimulate this area based on the results of previous experiments ${ }^{11,5-7}$. To stimulate the DLPFC, the coil was placed with the junction of the two coil wings above the target point. During the experiment the coil was fixed by means of a mechanical support.

\section{Cognitive Assessment}

Standard cognitive assessment was divided into two sessions. Neuropsychological testing was administered by an experienced examiner who was blind to patient treatment allocations. The cognitive assessment included tests to screen for dementia, together with 
neuropsychological tests for memory, executive functions and language. The results of the cognitive assessments at baseline, before rTMS treatment, and at two weeks (T2) are reported in Table 1 for both experimental groups. Moreover, we performed the same detailed cognitive assessment four weeks (T4) and 12 weeks (T12) after the onset of the rTMS treatment (note that T12 corresponds to 8 weeks after the end of the treatment). All the tests were administered and scored according to standard procedures ${ }^{12,13}$

Table 1

\section{Statistical analysis}

Demographic variables (age and education) of the two groups were compared at baseline, using parametric analyses (paired t-test). A p-value $<0.05$ was considered significant.

The behavioral effects induced by the rTMS protocol after two weeks of daily stimulation were assessed using a mixed-model ANOVA, considering group (Real-Real vs. Placebo-Real) as a between-subject factor, and time (baseline vs. two weeks) as a withinsubject factor. Further analysis was performed to assess the long-term efficacy of rTMS treatment using four time instants (Baseline vs. two weeks, four weeks and 12 weeks) as a within-subject factor.

\section{Results}

We identified no significant differences in the demographic variables between groups (Table 1, p>0.05).

To verify the presence of short-term behavioral effects induced by rTMS, we compared the performance of both groups at baseline and at the two-week evaluation. A significant difference between groups was found in terms of the percentage of correct responses only 
in auditory sentence comprehension subtest from the Battery for Analysis of Aphasic Deficits (SC-BADA) over time [group $x$ time interaction: $F(1,8)=6.07, p=.04 ; \eta_{p}^{2}=.43$ ]. The Real-Real group improved its performance $(p=.008)$ at two weeks $(77.3 \pm 6.5)$ with respect to baseline ( $66.6 \pm 8.6)$, whereas the Placebo-Real group showed no significant differences $(p=.99)$ between baseline $(66.0 \pm 7.1)$ and two weeks of placebo rTMS $(65.9, \pm 9.6)$.

We further analyzed SC-BADA scores at baseline, two, four, and 12 weeks to assess the long-term efficacy of rTMS in both groups. A significant main effect of time $[F(3$, $24)=3.87, p=.02]$ was found. Post-hoc analysis, Bonferroni, showed that the percentage of correct responses in SC-BADA at 12 weeks $(77.2 \pm 2.7)$ was still significantly different $(p=.02)$ from baseline $(66.3 \pm 7.45)$ (Figure 1B). No significant differences were found for other language abilities, nor for other cognitive functions (such as cognitive estimation and memory). See Table 1 for more details.

\section{Discussion}

The main purpose of this study was to investigate whether the application of highfrequency rTMS to the left DLPFC for 25 min a day, five days a week, for two weeks may lead to significant cognitive improvements in patients with AD. Specifically, we hypothesized that this protocol would result in changes in language performance, i.e., facilitation of language production and/or comprehension. In addition, we compared the effects of two or four weeks of stimulation to evaluate whether a longer rTMS application would result in a greater and/or longer-lasting effect. Finally, another important aim of the present study was to verify whether the cognitive benefits recorded immediately after rTMS treatment would persist eight weeks after the end of the treatment protocol (T12).

Overall, the results of our study show a significant effect of rTMS treatment on auditory sentence comprehension. 
In contrast with our previous studies ${ }^{5,6}$, in the present study we failed to observe a significant effect on naming performance in AD. These results may be attributed to the rTMS paradigm used (off-line vs. on-line) with a short term facilitation, in our previous study, strictly related to the timing of stimulation (i.e., few millisecond before the naming). In the present study we applied an off-line rTMS approach in which patients received daily rTMS treatment, while in the previous studies rTMS was applied to DLPFC during the execution of the naming task.

We also found that the administration of rTMS for four weeks did not result in additional improvements in performance compared to the application of rTMS for two weeks. A meta-analysis by the Cochrane Collaboration ${ }^{14}$ concluded that rTMS significantly improves depression only after a minimum of two weeks of treatment. Our results suggest that two weeks of rTMS is also sufficient to evidence behavioral improvements in $A D$ patients.

As regards the long-term effects, we identified an improvement in sentence comprehension eight weeks (T12) after the end of the rTMS intervention. To date, this is the first study that shows a long-lasting cognitive effect of rTMS treatments in AD patients.

Another important result of our study was the absence of any rTMS effects on memory and executive functions suggesting that learning effects cannot explain data. Therefore, the facilitation effect of DLPFC rTMS in AD appears to be specific to the language domain rather than reflecting a general, non-specific effect on cognitive processing.

Why did rTMS induce this improvement in patient language performance? The neurophysiological mechanisms responsible for rTMS-induced facilitation remain unknown. A number of investigations suggest that rhythmic transcranial stimulation can exert positive effects on cognitive performance ${ }^{4}$. A possibility is that the modification of cortical activity through the use of rhythmic stimulation may re-adjust pathological patterns 
of brain activity, thus providing an opportunity to induce new, healthier, activity patterns within the affected functional networks ${ }^{15}$.

The present findings may reflect an rTMS-induced modulation of short and/or longrange cortical synaptic efficacy and connectivity that potentiates the system within the language network, leading to more effective processing.

The present preliminary results highlight the therapeutic potential of the induction of long-term neuromodulatory effects using brain stimulation. They hold considerable promise, not only for advancing our understanding of brain plasticity mechanisms, but also for designing new rehabilitation strategies in patients with neurodegenerative disease. 
Table 1. Demographic and neuropsychological data. ${ }^{*}=p<.05$

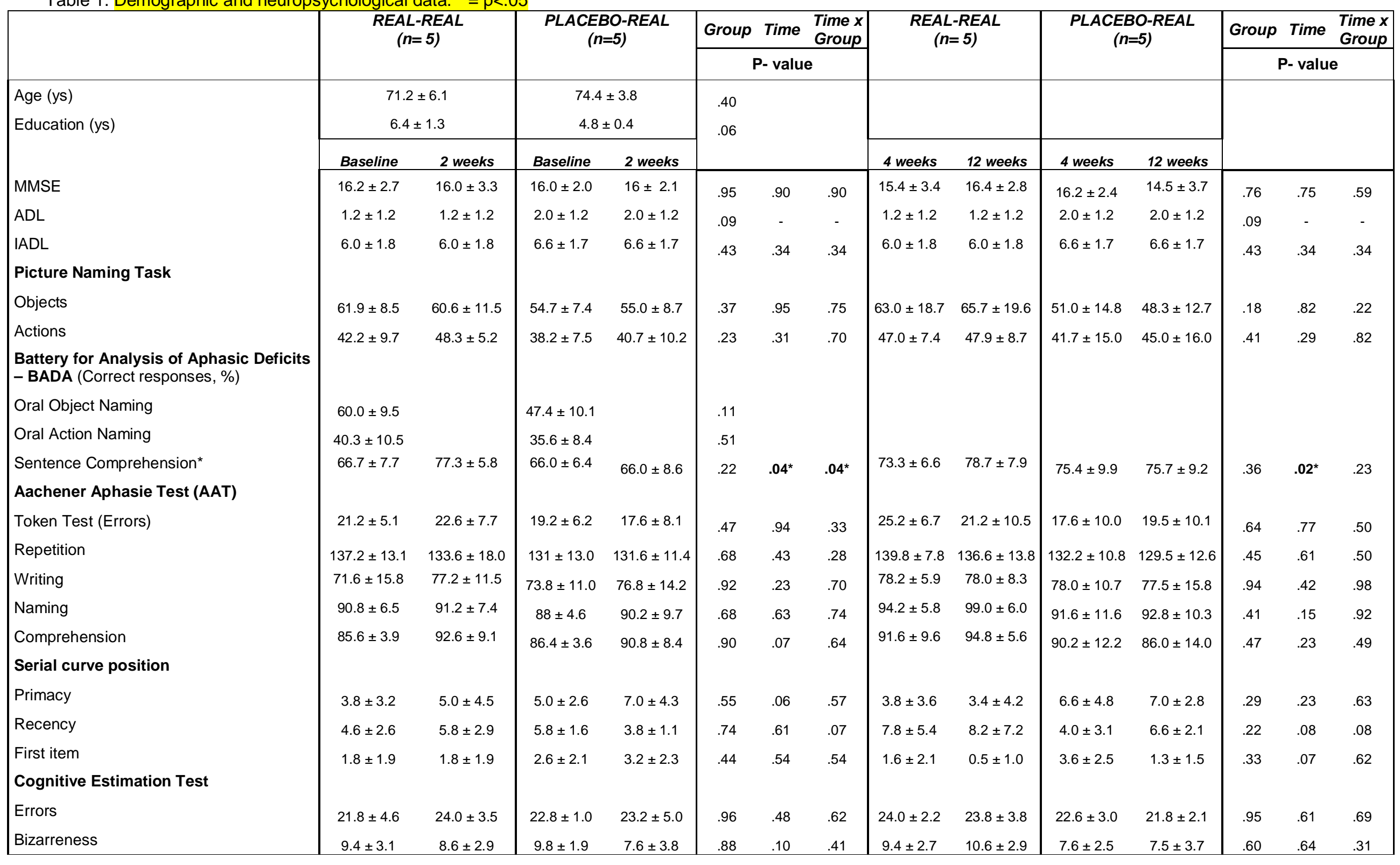




\section{Figure caption}

Figure 1.

Panel A Experimental paradigm. $A D=$ Alzheimer Disease, $R R=$ Real-Real treatment, $P R$ $=$ Placebo-Real treatment, $\mathrm{T} 2$ = assessment at two weeks from the baseline, $\mathrm{T} 4=$ assessment at four weeks from the baseline, $\mathrm{T} 12=$ assessment at twelve weeks from the baseline (i.e., eight weeks after rTMS treatment).

Panel B Comparison of percentage of correct responses on the auditory sentence comprehension subtest from the Battery for Analysis of Aphasic Deficits at T2, T4 and T12 between groups (Real-Real rTMS vs. Placebo-Real rTMS). T2 $=$ assessment at two weeks from the baseline, $\mathrm{T} 4=$ assessment at four weeks from the baseline, $\mathrm{T} 12=$ assessment at twelve weeks from the baseline (i.e., eight weeks after rTMS treatment). Means and standard errors are displayed. Asterisk indicates $p<.05$. 


\section{Acknowledgement}

We wish to thank patients and caregivers for their patience. This research was supported by a Project grant from the "Ministero della Sanità" and from Associazione Fatebenefratelli per la Ricerca (AFaR). 


\section{References}

1. Cotelli M, Calabria M, Zanetti O. Cognitive rehabilitation in Alzheimer's disease. Aging Clinical and Experimental Research 2006; 18: 141-3.

2. Warburton E, Price CJ, Swinburn K, et al. Mechanisms of recovery from aphasia: evidence from positron emission tomography studies. J Neurol Neurosurg Psychiatry 1999; 66: 155-61.

3. Buckner RL. Memory and executive function in aging and AD: multiple factors that cause decline and reserve factors that compensate. Neuron 2004; 44: 195-208.

4. Miniussi C, Cappa SF, Cohen LG, et al. Efficacy of repetitive transcranial magnetic stimulation/transcranial direct current stimulation in cognitive neurorehabilitation. Brain Stimulation 2008; 1: 326-36.

5. Cotelli M, Manenti R, Cappa SF, et al. Effect of transcranial magnetic stimulation on action naming in patients with Alzheimer disease. Archives of Neurology 2006; 63: 1602-4. 6. Cotelli M, Manenti R, Cappa SF, et al. Transcranial magnetic stimulation improves naming in Alzheimer disease patients at different stages of cognitive decline. European Journal of Neurology 2008; 15: 1286-92.

7. Manenti R, Cappa SF, Rossini PM, et al. The role of the prefrontal cortex in sentence comprehension: an rTMS study. Cortex 2008; 44: 337-44.

8. McKhann G, Drachman D, Folstein M, et al. Clinical diagnosis of Alzheimer's disease: report of the NINCDS-ADRDA Work Group under the auspices of Department of Health and Human Services Task Force on Alzheimer's Disease. Neurology 1984; 34: 939-44. 9. Rossi S, Hallett M, Rossini PM, et al. Safety, ethical considerations, and application guidelines for the use of transcranial magnetic stimulation in clinical practice and research. Clin Neurophysiol 2009; 120: 2008-39. 
10. Rossi S, Ferro M, Cincotta M, et al. A real electro-magnetic placebo (REMP) device for sham transcranial magnetic stimulation (TMS). Clin Neurophysiol 2007; 118: 709-16.

11. Cappa SF, Sandrini M, Rossini PM, et al. The role of the left frontal lobe in action naming: rTMS evidence. Neurology 2002; 59: 720-3.

12. Miceli G, Laudanna A, Burani C, et al. Batteria per l'Analisi dei Deficit Afasici. B.A.D.A. (Battery for Analysis of Aphasic Deficits). Milano: CEPSAG, Università Cattolica del Sacro Cuore, 1994

13. Lezak M, Howieson D, Loring DW. Neuropsychological Assessment (fourth edition). Oxford: University Press, 2004

14. Martin JLR, Barbanoj MJ, Schlaepfer TE, et al. Transcranial magnetic stimulation for treating depression. Cochrane Database of Systematic Reviews 2001; DOI:

10.1002/14651858.CD003493.

15. Thut G, Miniussi C. New insights into rhythmic brain activity from TMS-EEG studies. Trends Cogn Sci 2009; 13: 182-9. 


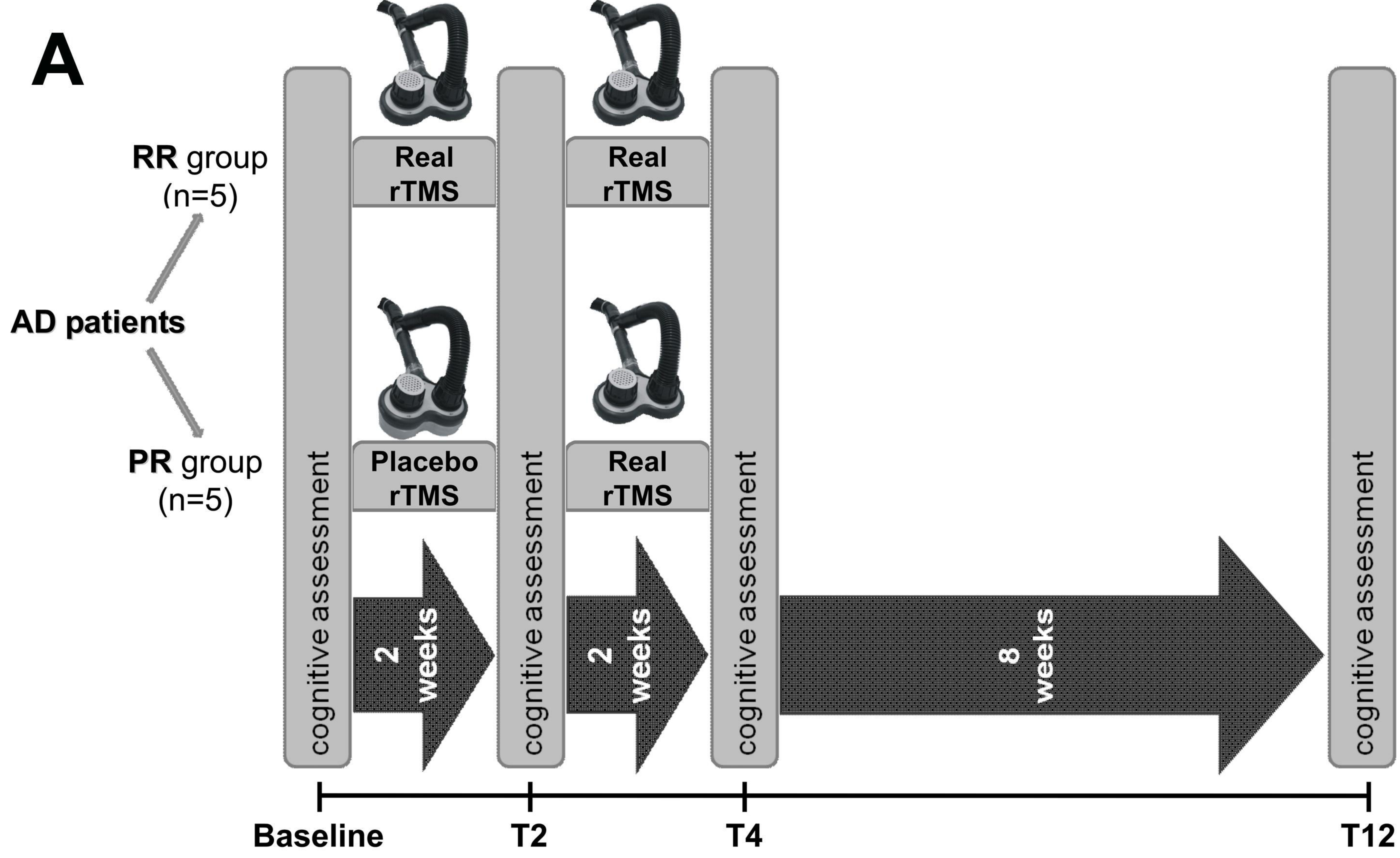

B

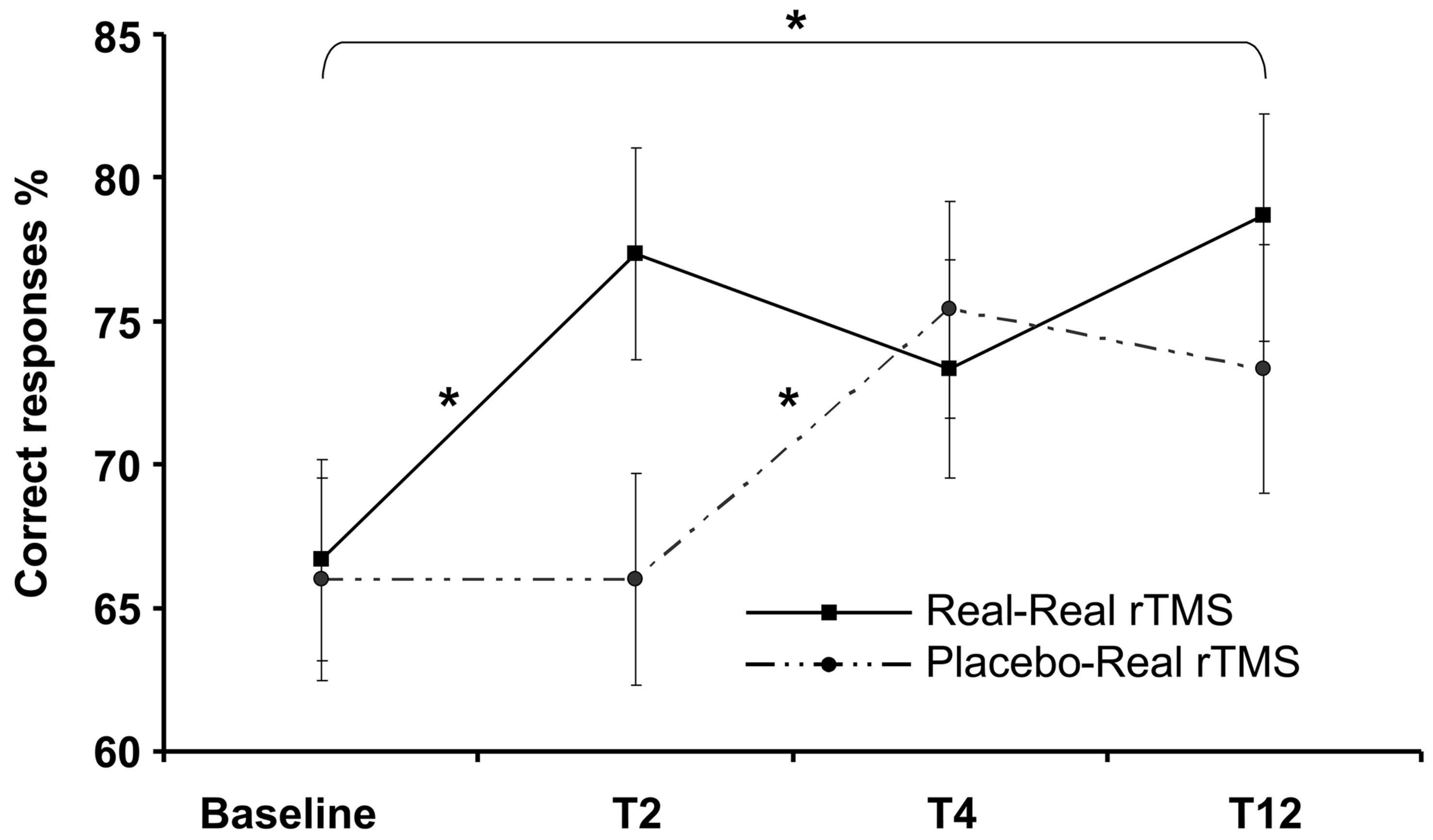

